\title{
Adenomatous polyp at the ureterocaecal anastomosis
}

\author{
L.D. Coen and A.T. Raftery
}

Sheffield Kidney Institute, Northern General Hospital, Herries Road, Sheffield S5 7AU, UK

\begin{abstract}
Summary: A case of adenomatous polyp occurring at a ureterocaecal anastomosis, 25 years after urinary diversion following a total cystectomy for carcinoma of the bladder, is reported. Bilateral nephrectomy for chronic pyelonephritis was carried out 25 years after the initial surgery and, following this, a sinus formed at the incision at the right loin. A sinogram showed contrast filling the right ureter and caecum, and outlined a lobulated filling defect at the ureterocaecal anastomosis. Subsequent histology revealed a dysplastic tubulovillous adenoma. The clinical presentation and management of tumours at the ureterointestinal junction are discussed.
\end{abstract}

\section{Introduction}

Tumours at the ureterointestinal junction are a known complication of urinary diversion into the bowel. Hammer reported the first case in $1929,{ }^{1}$ although fewer than 100 cases have since been described. We report a further case which is notable for its unusual presentation.

\section{Case report}

At the age of 48 a Caucasian female underwent a total cystectomy and Wertheim's hysterectomy for a poorly differentiated transitional cell carcinoma infiltrating the bladder wall. The left ureter was anastomosed to the sigmoid colon and the right one to the caecum.

Over the ensuing 25 years the patient went on to develop chronic pyelonephritis suffering recurrent episodes of septicaemia with associated faecal incontinence. The urinary diversion was no longer sufficient to maintain adequate renal function and, at the age of 74 , she underwent bilateral extraperitoneal nephrectomy through separate loin incisions.

She was subsequently referred to our unit for vascular access for haemodialysis. One month after nephrectomy, she developed a purulent discharge from the right loin associated with rigors and the passage of dark blood per rectum. Examination revealed a possible abdominal mass underlying the discharging sinus, a suggestion supported by abdominal ultrasonography. A sinogram demonstrated a cavity lateral to the bodies of second and third lumbar vertebrae with contrast filling the right ureter and caecum. A lobulated filling defect

Correspondence: A.T. Raftery, M.D., F.R.C.S. Accepted: 30 October 1993 could be seen at the ureterocaecal junction suggesting the presence of a tumour (Figure 1).

The patient proceeded to laparotomy and a right hemicolectomy with en bloc resection of the ascending colon and ureteric stump, and drainage of the paracolic abscess was performed.

A pedunculated polyp measuring $5 \times 4 \times 3 \mathrm{~cm}$ was found at the ureterocaecal junction (Figure 2). Histology of the polyp showed a moderately differentiated dysplastic tubulovillous adenoma. The patient made a slow recovery and, at 6 weeks, is dialysing satisfactorily via the graft in the right thigh. Postoperative colonoscopy failed to reveal any other abnormality in the remaining large bowel.

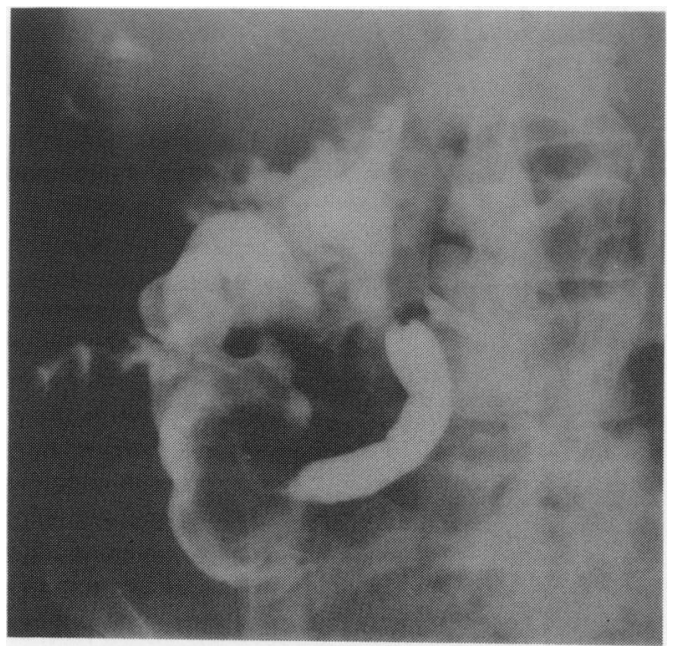

Figure 1 Sinogram showing lobulated filling defect in the caecum. The ureter and the abscess cavity are outlined by the contrast. 


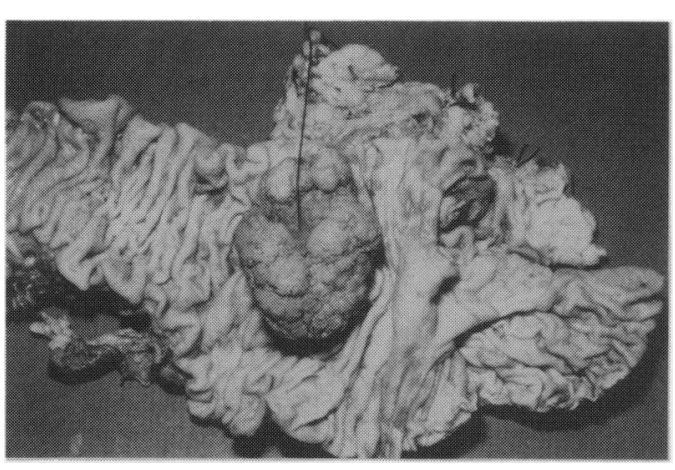

Figure 2 Operative specimen showing a polyp at the ureterocaecal junction. A probe is shown passing through the ureteric orifice.

\section{Discussion}

Ninety-four cases of tumours at the site of ureterocolic anastomosis ${ }^{2}$ and four cases of tumours arising in isolated colon ${ }^{3,4}$ or ileal ${ }^{5,6}$ loops have been described following urinary diversion. Eighty-four cases were originally treated for benign conditions whilst 14 had underlying malignant disease. Altogether, 27 benign polyps have been reported, 24 occurring in patients with original benign pathology. Our case is only the fourth one where a benign polyp has been found in a patient treated by ureteroenterostomy for primary malignant disease. This case is unique for its clinical presentation following the cure of a particularly aggressive primary tumour.

The risk of a tumour developing at the site of a ureterocolic anastomosis has been reported to lie between 100 and 500 times that of the general population ${ }^{2,3,7}$ with up to $40 \%$ of cases developing tumours. ${ }^{8}$ The mean age at the time of tumour diagnosis shows two distinct groups, reflecting whether the initial diversion was carried out for benign or malignant disease and being 35 and 61 years, respectively. ${ }^{2,9}$ The overall mean latent period of tumour development is 25 years, but only 10 years for patients originally treated for malignant disease. $2,7,9$ These tumours tend to arise around poorly or non-functioning ureteroenteric anastomoses. ${ }^{2}$ The clinical manifestations are alteration of bowel habit with rectal bleeding ${ }^{9}$ and constipation, abdominal pain and urinary symptoms. ${ }^{2}$ Diagnosis presents difficulties particularly in differentiating tumour from a prolapsing ureter surrounded by chronic inflammatory reaction. ${ }^{9}$ Recommended investigations include endoscopy and biopsy, intravenous urogram and contrast studies of the bowel. ${ }^{10}$ Correlating the radiographic findings is often necessary to reach a diagnosis. ${ }^{10}$

Treatment requires resection of the tumour containing segment of bowel with complete removal of the ureteric stumps ${ }^{9}$ and fashioning of an external conduit or reimplantation of the ureters into the bowel. In our case, failed excision of the ureter contributed to the clinical picture and to the delay in treatment. Regular endoscopic follow-up as well as urine culture and intravenous urography are recommended. ${ }^{11}$

The problem of tumours of the ureterointestinal anastomosis does not seem to be confined to internal urinary diversions. ${ }^{3-6}$ External diversions were first described and popularized between 1950 and 1960 by Bricker ${ }^{12}$ and Mogg. ${ }^{13}$ Taking into account the long period of latency for the development of such tumours we could expect an increase in rate of this complication in the future.

\section{References}

1. Hammer, E. Cander du colon sigmoide dix ans apres implantation de ureteres d'une vessie exstrophie. $J$ Urol (Paris) 1929, 28, 260-263.

2. Stewart, M. Urinary diversion and bowel cancer. Ann R Coll Surg Engl 1986, 68: 98-102.

3. Wilson, J.W.L. \& Morales, A. Development of adenocarcinoma in transverse colon conduit. Urology 1982, 20: $182-183$.

4. Chiang, K.S., Minton, J.P., Clausen, K., Clatworthy, H.W. \& Wise, H.A. Carcinoma in a colon conduit urinary diversion. $J$ Urol 1982, 127: 1185-1187.

5. Tomera, K.M., Unni, K.K. \& Utz, D.C. Adenomatous polyp in ileal conduit. $J$ Urol 1982, 128: 1025-1026.

6. Shousa, S., Scott, J. \& Polak, J. Ileal loop carcinoma after cystectomy for bladder exstrophy. $\mathrm{Br} \mathrm{Med} J$ 1978, 2: 397-398.

7. Crissey, M.M., Steele, G.D. \& Gittes, R.F. Rat model carcinogenesis in ureterosigmoidostomy. Science 1981, 214: 1079-1080.

8. Cohen, M.S., Hilz, M.E., Davis, C.P. \& Anderson, M.D. Urinary carcinogen [nitrosamine] production in a rat animal model for ureterosigmoidostomy. J Urol 1987, 138: 449-452.

9. Rivard, J.Y., Bedard, A. \& Dionne, L. Colonic neoplasms following ureterosigmoidostomy. J Urol 1975, 113: 781 - 786 .

10. Princenthal, R.A., Lowman, R., Zeman, R.K. \& Burrell, M. Ureterosigmoidostomy: the development of tumours, diagnosis, and pitfalls. $A J R$ 1983, 141: 77-81.

11. Moorcraft, J., DuBoulay, C.E.H., Isaacson, P. \& Atwell, J.D. Changes in the mucosa of colon conduits with particular reference to the risk of malignant change. Br J Urol 1983, 55: 185-188.

12. Bricker, E.M. Bladder substitution after pelvic evisceration. Surg Clin North Am 1950, 30: 1511-1521.

13. Mogg, R.A. The treatment of neurogenic urinary incontinence using the colonic conduit. J Urol 1965, 37: 681-686. 